\title{
Comparative evaluations of the processed bovine tunica vaginalis implant in a rat model
}

\begin{abstract}
The aim of the present study was to evaluate the lyophilized and glycerolized bovine parietal tunica vaginalis for repair of a full-thickness abdominal wall defect and to compare their effectiveness with expanded polytetrafluoroethylene (ePTFE) Mycro Mesh (Gore-Tex ${ }^{\circledR}$ MYCRO MESH®; Gore and Designs, W. L. Gore \& Associates, Flagstaff, AZ, USA) in a rat model. Fresh bovine parietal tunica vaginalis sacs collected from an abattoir were processed and preserved by freeze-drying and by using $99.5 \%$ glycerol. Full-thickness abdominal wall defects $(3 \times 2.5 \mathrm{~cm})$ created surgically in 90 male Sprague-Dewaly rats $(300-400 \mathrm{~g})$ were repaired with the same size of lyophilized, glycerolized or ePTFE Mycro Mesh with 30 rats in each group. Six rats from each group were killed at post-implantation intervals of 1, 3, 6, 9 and 18 weeks for macroscopic, microscopic and tensiometric evaluations. All rats survived the procedure, except for one rat in the ePTFE Mycro Mesh group. Implants of bovine origin were gradually resorbed and replaced with recipient fibrous tissue, whereas the mesh implant was encapsulated with fibrous tissue and remained without any marked changes throughout the study period. Adhesions between the implant and underlying visceral organs were encountered in 10, 6.6 and $3.3 \%$ of rats implanted with lyophilized, glycerolized or ePTFE Mycro Mesh, respectively. Foreign body giant cells and calcification were demonstrated in fibrous capsule and mesh matrix, respectively, in rats implanted with ePTFE Mycro Mesh. Neither of these characteristics were observed in rats implanted with processed bovine tunica vaginalis. Macrophages engorged with lipofuchsin pigments were observed in the recipient tissue that replaced the implants of bovine origin at 3-18 weeks post-implantation. There were no significant $(\mathrm{P}>0.05)$ differences in total mean values of healing tensile strength, load at break and Young's modulus of elasticity between the three implant groups. The results of the present study encourage further investigation into the use of processed bovine parietal tunica vaginalis in clinical practice.
\end{abstract}

Keyword: abdominal defect, bovine tunica vaginalis, glycerol, healing strength 